Rochester Institute of Technology

RIT Scholar Works

Spring 5-8-2021

\title{
2VT: Visions, Technologies, and Visions of Technologies for Understanding Human Scale Spaces
}

\author{
Ville Paanen \\ University of Oulu \\ Piia Markkanen \\ University of Oulu \\ Jonas Oppenlaender \\ University of Oulu \\ Haider Akmal \\ University of Lancaster \\ Lik Hang Lee \\ Korea Advanced Institute of Science \& Technology
}

See next page for additional authors

Follow this and additional works at: https://scholarworks.rit.edu/other

Part of the Other Computer Sciences Commons

\section{Recommended Citation}

Ville Paananen, Piia Markkanen, Jonas Oppenlaender, Lik Hang Lee, Haider Akmal, Ava Fatah gen. Schieck, John Dunham, Konstantinos Papangelis, Nicolas Lalone, Niels van Berkel, Jorge Goncalves, and Simo Hosio. 2021. 2VT: Visions, Technologies, and Visions of Technologies for Understanding Human Scale Spaces Authors copy. In CHI Conference on Human Factors in Computing Systems Extended Abstracts (CHI '21 Extended Abstracts), May 8-13, 2021, Yokohama, Japan. ACM, New York, NY, USA, 9 pages. https: //doi.org/10.1145/3411763.3441315

This Conference Paper is brought to you for free and open access by the Faculty \& Staff Scholarship at RIT Scholar Works. It has been accepted for inclusion in Presentations and other scholarship by an authorized administrator of RIT Scholar Works. For more information, please contact ritscholarworks@rit.edu. 


\section{Authors}

Ville Paanen, Piia Markkanen, Jonas Oppenlaender, Haider Akmal, Lik Hang Lee, Ava Fatah Gen Schieck, John Dunham, Konstantinos Papangelis, Nicolas Lalone, Niels Van Berkel, Jorge Goncalves, and Simo Hosio 


\section{VT: Visions, Technologies, and Visions of Technologies for Understanding Human Scale Spaces Authors copy}

VILLE PAANANEN, University of Oulu, Finland

PIIA MARKKANEN, University of Oulu, Finland

JONAS OPPENLAENDER, University of Oulu, Finland

LIK HANG LEE, Korea Advanced Institute of Science and Technology, South Korea

HAIDER AKMAL, Lancaster University, United Kingdom

AVA FATAH GEN. SCHIECK, University College London, United Kingdom

JOHN DUNHAM, Rochester Institute of Technology, USA

KONSTANTINOS PAPANGELIS, Rochester Institute of Technology, USA

NICOLAS LALONE, University of Nebraska, USA

NIELS VAN BERKEL, Aalborg University, Denmark

JORGE GONCALVES, University of Melbourne, Australia

SIMO HOSIO, University of Oulu, Finland

Spatial experience is an important subject in various fields, and in $\mathrm{HCI}$ it has been mostly investigated in the urban scale. Research on human scale spaces has focused mostly on the personal meaning or aesthetic and embodied experiences in the space. Further, spatial experience is increasingly topical in envisioning how to build and interact with technologies in our everyday lived environments, particularly in so-called smart cities. This workshop brings researchers and practitioners from diverse fields to collaboratively discover new ways to understand and capture human scale spatial experience and envision its implications to future technological and creative developments in our habitats. Using a speculative design approach, we sketch concrete solutions that could help to better capture critical features of human scale spaces and allow for unique possibilities for aspects such as urban play. As a result, we hope to contribute a road map for future $\mathrm{HCI}$ research on human scale spatial experience and its application.

CCS Concepts: • Human-centered computing $\rightarrow$ HCI theory, concepts and models; • Applied computing $\rightarrow$ Architecture (buildings).

Additional Key Words and Phrases: spatial experiences, speculative design, human-building interaction

\section{ACM Reference Format:}

Ville Paananen, Piia Markkanen, Jonas Oppenlaender, Lik Hang Lee, Haider Akmal, Ava Fatah gen. Schieck, John Dunham, Konstantinos Papangelis, Nicolas Lalone, Niels van Berkel, Jorge Goncalves, and Simo Hosio. 2021. 2VT: Visions, Technologies, and Visions of Technologies for Understanding Human Scale Spaces Authors copy. In CHI Conference on Human Factors in Computing Systems Extended Abstracts (CHI '21 Extended Abstracts), May 8-13, 2021, Yokohama, Japan. ACM, New York, NY, USA, 9 pages. https: //doi.org/10.1145/3411763.3441315

Permission to make digital or hard copies of part or all of this work for personal or classroom use is granted without fee provided that copies are not made or distributed for profit or commercial advantage and that copies bear this notice and the full citation on the first page. Copyrights for third-party components of this work must be honored. For all other uses, contact the owner/author(s).

(c) 2021 Copyright held by the owner/author(s).

Manuscript submitted to ACM 


\section{INTRODUCTION}

The subject of spatial experience is pivotal in HCI and relevant other fields, such as architecture, urban design, geography, and philosophy. For the same reason, it can be approached through various different analytical lenses [5]. For instance, the traditional Cartesian understanding of spaces, i.e. the geometric coordinate system, is beneficial for constructing buildings, but does not account for the multitude of spatially formed behaviors that are critical in HCI, e.g. dwelling, interacting, or encountering [11,14]. In addition to the Cartesian understanding, spaces can also be understood through their attributes, such as structural properties, form and design, or other metrics such as personal meaning and functional affordances or lack thereof. As a result, explication of spaces also requires analysis of the subjective constituents [12]. This workshop seeks to move the field of HCI one step forward in terms of our collective understanding of spatial experience in human scale.

Humans innately experience their surrounding environments from their own, human, scale [7]. The visual experience is often mediated on eye level. The experience differs in relation to the surrounding scale, for example, a street as opposed to a motorway, or a room as opposed to a skyscraper. Human scale environments are inherently important in HCI, as the de facto contexts of use for most applications and spaces where creative installations are deployed. In this context, in our built environments, the proliferation of embedded technologies necessitates new methods of understanding spaces, as their complexity is rapidly growing beyond what can be accounted for by contemporary HCI methods $[1,14,16]$.

One of the contexts where this development is evident is in the design of our future living environments: "[in] the rush to create so-called 'smart cities'... much of the attention has been on how to technically create and implement suitable smart city technology" [15]. Further, the COVID-19 pandemic has pointed to weaknesses and assumptions about technologies for the everyday life of the city. It has also pointed to vulnerable populations and populations that fall outside of even basic messaging. Thus, for instance, the need for distributing information across a city while protecting citizens from misinformation has become an issue that alters the meaning of these smart spaces. By unpacking these assumptions, designers and researchers gain new avenues, new ways to think about how to design the spaces in the midst of local, regional, national, and international crisis events. An important aspect of those assumptions is a deeper engagement with the lived experience of 'smart spaces' that explores the extent to which and for whom these physical and digital environments are currently producing new forms of subjective experiences, e.g. play and playfulness [17].

This interdisciplinary workshop brings together scientists and practitioners interested in spatial experience from the fields of technology, design, architecture, and creative arts, to improve our collective understanding of human scale spaces and its broad-ranging future applications. We start by seeking common ground on which spatial features can best convey the holistic understanding of a human scale space. We then speculate on technologies that could be used to capture these features and seek to envision the potential implications of such added spatial understanding to different disruptive future technologies and creative undertakings, such as in cases of locative games or urban play.

We are confident this workshop will attract participants with diverse backgrounds and would contribute to CHI 2021 with a melting pot of different ideas on the notion of human scale spaces. The implications of an increased understanding of how to model human scale spaces would be relevant to various different domains considered pivotal in HCI, including mobile applications, IoT, situated technologies, virtual and augmented reality, computational architecture, spatial arts, and boundary-crossing specialized applications that intersect these domains. Ultimately, we aim for a future research road map to provide a harmonized way of thinking about human scale spaces and their applications especially in the context of HCI. 


\subsection{Prior Art}

Most previous work has investigated methods for spatial understanding in the scope of urban environment, cities, and regional areas $[3,13]$. For instance, web-based crowdsourcing has been shown as efficient in characterizing neighborhoods by deriving spatial features from user-generated content, both online [13] and offline [20]. More related to the human scale, in the pair of seminal papers on spaces and places in HCI $[5,10]$, the researchers focused on the understanding of humans' spatial dimensions and how the concepts of space and place could be used in design. Since the publication of these works, technological innovations have grown in number and their effect, and spatiality is now an important consideration in e.g. virtual reality [18] or even IoT [4]. Thus, there is a risk that the understanding of the embodied human experience cannot keep up with the fast-paced technology development.

Krogh et al. [16] used the theory of proxemics, the study of human use of space [9], to form conceptual tools for the design process. The current technologies pose limitations to the human spatial behaviors, as exemplified by the limitation of the tracking algorithm of Kinect when the tracking skeleton overlaps with another. Introducing the understanding of spatial behaviors to the field of HCI allows interaction designers to form more spatially aware decisions. In a similar manner, Kirsh [14] discusses the notions of interaction and interfaces in relation to HCI and architecture. The differences run deep: "People don't read buildings the way they do HCI interfaces" [14]. However, Kirsh [14] gives a detailed description on how to approach spatiality in these fields; the limitations of traditional interaction patterns in terms of agency, intention, and control, serve as a strong argument that we need to frame our thinking in a new way when thinking about spatiality in HCI. To tackle this task of developing new understanding, the emerging notion of Human-Building Interaction, or HBI, is focusing on ways to connect the built environment closer to the field of HCI. The goal of HBI is to form new understanding for future technologies, and to "provide a framework that can be used to understand, compare and relate the converging research efforts from the two fields of HCI and Architecture in envisioning and shaping the future of living. [1].

As the topic of our workshop is not an established facet of HCI, it is advantageous to form an understanding of the possibilities of spatial experience for technology development. To tackle this, we will use Speculative Design [6] to envision the possibilities of spatial experience in HCI. Speculative Design has been used to explore possible future scenarios, and to aid the development of new technologies. For example, Stals et al. [19] used Speculative Design to investigate people's emotional relationships with places in urban environments. The use of Speculative Design has been criticized for the biased outcomes, due to the privileged positions of the designers [8]. In 2018, a symposium on 'The Secret Life of Buildings' sought to explore how object-oriented ontology could influence architectural theory[2]. However, in the case of this workshop, the subject matter of spatial experiences is universal and personal, which gives a lot of fuel to use a method like Speculative Design, without being critically biased.

\subsection{Objectives of this Workshop}

The overarching principle of our workshop to provide an international agora for practitioners from various backgrounds to be exposed to existing discussions and theories of space, stimulate multidisciplinary discussion, and explore issues that attend to human scale spaces in the smart city, explicit and implicit forms of play facilitated by the smart city, and the possibilities provided by new technologies. Our main objectives are as follows:

(1) Multidisciplinary discussion to elucidate common cross-disciplinary and distinctive discipline-specific spatial features to build shared understanding on the actual lived, human scale spatial environment. We aim to arch understanding of attributes over disciplines and viewpoints, for example from forming new possibilities for play 
in smart cities or inspecting realism enhancing attributes needed in VR development into capturing the 'digital soul of a space' in creative arts.

(2) Speculative design of future technologies, approaches, and methods enable the capture and collection of identified features in a machine-storable format. Such a format is needed for the features to be better used as a building block in the context of HCI and digital solutions.

(3) Sketch out a road map with short and long term objectives for future research of human scale spatial understanding and mapping out potential implications of such increased understanding of spatial experience for future disruptive technologies, such as XR, smart cities, Digital Twins, Smartphone Applications, crowdsourcing solutions, locative games, etc.

At the end of the workshop, we will summarize our findings and design speculations on the workshop website. Further, we plan to publish a joint article on the results from the workshop, in a relevant multidisciplinary journal.

\section{PRE-WORKSHOP PLANS}

We will set up the workshop home page online, using the website of the Crowd Computing Research Group as the host site: https://crowdcomputing.net/2vt-workshop. At first, the domain acts simply as a creative front for the workshop that contains an extended version of the Call for Papers along with all the relevant dates and submission information. Additionally, we will include some motivational bits and pieces of the organizers' own work in this domain, to foster imagination.

Following the publication of the website, we will distribute the CfP via relevant mailing lists but also through the extensive colleague networks of the organizing committee, across several different organizations and continents. With respect to involving practitioners in this workshop, we will draw upon our connections with developers at organizations such as Microsoft, Google, and Niantic Labs, several of whom regularly attend CHI. Further, to engage with participants that do not traditionally attend $\mathrm{HCI}$ conferences we will leverage our contacts and invite developers, digital media organizations, memory institutions, policymakers, designers, and others who may be interested. After the organizing committee has selected the participants, they are instructed to record a short video of them exploring their urban environment. The video will be uploaded to a video hosting site, such as YouTube, as an unlisted video. The pre-recorded urban exploration will be used in the latter half of the workshop as a way to test the participants' ideas in various environments.

\section{WORKSHOP STRUCTURE}

We aim for between 15 to 20 participants (excluding organizers) with multidisciplinary backgrounds, who will work in small groups during the workshop on themes most relevant to their submissions. The workshop will take place on Zoom. We will begin the day with an introduction to the topic of the workshop and the organizers' presentation. Next, the workshop participants introduce themselves, after which we will group them together based on the topic of their submissions. The participants will then present their workshop submissions informally in their groups. After the introductions of the organizers and participants, and the informal submission presentations, we will have a coffee break.

Next, as the first task of the workshop, the participants will be given a Speculative Design task to come up with ways of gathering and applying spatial understanding. The participants are instructed to focus on a single scenario where the human spatial experience and the surrounding space are in interaction, and to visualize it, for example, a case of urban play in smart cities. Afterwards, each participant is given 3 minutes to present their ideas. During the 
workshop, we use a shared collaboration tool online (e.g., Padlet, Miroboard) where all the photos and writings will be recorded, starting with the first design speculations.

After a coffee break, the participants will be divided into the previously formed breakout groups (2-4 participants in each) to focus on different fields where the increased understanding of human experience in spaces will have implications; What and how exactly? The groups are free to bring their own expertise to the ideation process, but the organizers also provide inspiration through demonstrating Speculative Design and Design Fiction methods to support the group work. Like previously, groups will document their findings and designs on the shared collaboration tool online. The morning session will conclude with a lunch break.

The afternoon session will start after lunch by utilizing participants' pre-recorded urban explorations around the participants' own neighborhoods, which allows the participants to gain further inspiration and are able to contrast their thinking by overlaying it to the physical surroundings under their respective themes. This approach allows the participants to refine and validate their ideas in a real-world context. Further, the pre-recorded explorations provide a diverse set of environments, based on the participants' locations.

Following the urban exploration, we will continue working in the groups in the online platform and the participants will further develop their ideas. We will specifically ask them to consider the implications of their ideas in regards to technology development. As the final task for the participants, they will present their groups' results. The workshop will conclude by reflecting on the outcomes of the day, led by the organizers.

First Session

\begin{tabular}{l|l}
\hline $30 \mathrm{~min}$ & Workshop introduction and introductions \\
$15 \mathrm{~min}$ & Informal submission presentations in groups \\
$30 \mathrm{~min}$ & Speculative Design task individually \\
$30 \mathrm{~min}$ & Coffee break \\
$60 \mathrm{~min}$ & Further technology speculation in groups \\
$45 \mathrm{~min}$ & Lunch break \\
\multicolumn{2}{|c}{ Second Session } \\
\hline $60 \mathrm{~min}$ & Urban exploration to validate technology groups' ideas \\
$30 \mathrm{~min}$ & Coffee break \\
$30 \mathrm{~min}$ & Implications, working in groups \\
$15 \mathrm{~min}$ & Small group presentations \\
$15 \mathrm{~min}$ & Reflection, closing the workshop
\end{tabular}

\section{POST-WORKSHOP PLANS}

After the workshop, we will publish a concise summary of the resulting design speculations as well as the future research ideas in form of a road map on the workshop website. More importantly, we seek to publish a joint article that summarises the results with a polished road map, with all voluntary workshop participants in a suitable special issue, optimally in a multidisciplinary journal that is welcoming to work that intersects $\mathrm{HCI}$ and architecture, such as Frontiers of Architectural Research (ISSN 2095-2635) and TOCHI (Transactions on Computer-Human Interaction, ISSN 1073-0516). 


\section{WEBSITE}

The workshops website can be found at https://crowdcomputing.net/2vt-workshop. We will primarily use the website to:

- advertise the workshop,

- communicate information to the participants, and host information about the participants,

- disseminate the workshop outcomes, including all presented materials.

\section{ORGANIZERS}

As this workshop is intended for a diverse audience of technology experts, architects, creative designers, and artists alike, we have gathered an organizing committee that is well-suited to support as well as publicize the workshop to ensure participation. The committee is also experienced in organizing CHI workshops and, more recently, hybrid and virtual conferences.

Ville Paananen is a doctoral student at the Center for Ubiquitous Computing, University of Oulu, Finland. His research interests lie in the cross-section of $\mathrm{HCI}$ and architecture, the emergent notion of Human-Building Interaction, and how the future technologies can be more spatially aware.

Piia Markkanen is a doctoral student in Oulu School of Architecture, University of Oulu, Finland. Her current research focuses on evaluating situation-related spatial experiences in work environments. She uses user-centric design methods and real-world intervention studies in her research to explore different spatial dimensions (e.g. functional, symbolic, aesthetic) and elements, such as lighting and acoustics, that holistically influence spatial experiences.

Jonas Oppenlaender is a final-year doctoral student and a member of the Crowd Computing research group at the Center for Ubiquitous Computing, University of Oulu, Finland. His research interests include crowdsourcing, crowd feedback systems, and leveraging mixed-initiative systems for engaging, supporting, and empowering humans.

Lik Hang Lee is an Assistant Professor at the Korea Advanced Institute of Science and Technology (KAIST), Daejeon, South Korea. His research interests include augmented reality (AR) and virtual reality (VR). His recent research on AR/VR has investigated how to achieve city-wide user experience with such enriched contents and make the user-centric coexistence between digital entities and physical counterparts in an emerging form of spatiality.

Haider Akmal is a final-year PhD candidate at Lancaster University, UK and part of Imagination Lancaster and Uncanny AI. With a keen focus on Research through Design, Ludic Design, and Design Fiction his work challenges established orthodoxies for the Design of IoT by exploring More-than Human Design Futures through speculative design research, play, and philosophy to forge untapped technological opportunities in HCI.

Ava Fatah gen Schieck is a Researcher, Educator and Architect. She is Associate Professor in Media Architecture and Urban Digital Interaction at the Bartlett, University College London. The main focus of Ava's research is in the area of Architecture, Interaction Design, and Ubiquitous Computing (AR, VR, and XR). Her research is practice based where she investigates the design, integration and evaluation of digital and physical ecologies within various environments and across different scales of interaction, from the body scale into the city scale. She is the Principal investigator of 'Screens in the wild', resulting in a unique 'living lab', environment of four interactive networked screens in London and Nottingham (UK). 
John Dunham is a PhD student in Computing and Information Sciences at Rochester Institute of Technology and a member of the Niantic x RIT Geo Games and Media Lab. John completed his M.Sc. in Game Design and Development at Rochester Institute of Technology. His current work focuses on location-based games and their impact on health and wellbeing.

Konstantinos Papangelis is an Assistant Professor at the School of Interactive Games and Media of Rochester Institute of Technology, and the director of the Niantic x RIT Geo Games and Media Lab (https://www.rit.edu/NianticGeoGamesLab). His research currently focuses on location-based games and social networks, the physical web, location-based and in-situ crowdsourcing, proximity technologies, extended and hybrid reality, and multi-sensory entertainment technologies.

Nicolas Lalone is an Assistant Professor at the University of Nebraska at Omaha. As a researcher, Dr. LaLone's work focuses on disrupted spaces such as those within a crisis event like an earthquake, riot, or hurricane. He is actively pursuing new ways to train to use, design, and approach the evaluation of computational products that may have a use in saving lives.

Niels van Berkel is an Assistant Professor at the Human-Centered Computing Group at Aalborg University. His research interests lay in Human-Computer Interaction, Social Computing, and Ubiquitous Computing. On the topic of spatiality, he has studied the use of contextual data collection methods (e.g., crowdsourcing, citizen science) to support the creation of structured and unstructured local knowledge bases.

Jorge Goncalves is a Senior Lecturer in Human-Computer Interaction at the School of Computing and Information Systems in the University of Melbourne. His interests are in crowdsourcing, situated technologies, and Social Computing. Goncalves is a pioneer of situated crowdsourcing, including work on enabling situated technologies to self-learn about the surrounding space as a byproduct of offering gamified experiences to passersby.

Simo Hosio is an Associate Professor at the Center for Ubiquitous Computing, University of Oulu, Finland. He leads the Crowd Computing Research Group and is also associated with the Center for Life Course Health Research. His research interests include social computing, crowdsourcing, ubiquitous computing.

\section{CALL FOR PARTICIPATION}

Spatial understanding refers to the perceptions, measurements, and representation of our surroundings. How will the future technologies, applications, or creative installations leverage such spatial understanding? This one-day workshop calls for experts from all related fields to ideate and contribute to our collective understanding and application of spatial experience.

This workshop is interested in the human scale: eye-level environments that are the de facto operating environment for most end-user technologies. We are open to a broad audience to stimulate the workshop participants by exposure to new points of view from different disciplines.

We invite your submissions on how different future technologies might benefit from increased spatial understanding. There are two submission types that we encourage:

(1) a "traditional" paper providing a scientific contribution with empirical results, or 
(2) a "reflection" to propose visions or concepts and to reflect on how human scale spatial experience affects technological or creative solutions.

We encourage you to communicate your ideas using a variety of methods: text, images, photos, sketches, and more. The submissions should use the DIS2020 Pictorial Format with a length of 4-6 pages (excluding references). Further details can be found at https://crowdcomputing.net/2vt-workshop. We accept your submissions as a pdf by February 19 th, 2021. At least one author of each accepted submission paper must attend the workshop and all participants must register for both the workshop and for at least one day of the conference. Participants will be selected by a juried process, based on their experience, expressed interest, and the content of the submission. To gather a rich set of views, we will focus on the diversity of the group of participants.

\section{Important Workshop Dates}

Call for papers: December 15th, 2020

Submission Deadline: February 19th, 2021

Notification of acceptance: February 28th, 2021

Workshop Date: May 8th, 2021

\section{REFERENCES}

[1] Hamed S. Alavi, Elizabeth F. Churchill, Mikael Wiberg, Denis Lalanne, Peter Dalsgaard, Ava Fatah Gen Schieck, and Yvonne Rogers. 2019. Introduction to human-building interaction (HBI): Interfacing HCI with architecture and urban design. ACM Transactions on Computer-Human Interaction 26, 2 , Article 6 (mar 2019), 10 pages. https://doi.org/10.1145/3309714

[2] Michael Benedikt and Kory Bieg. 2018. CENTER 21: The Secret Life of Buildings. Center for American Architecture and Design, Austin, Texas, USA.

[3] Olga Chesnokova, Mario Nowak, and Ross S. Purves. 2017. A Crowdsourced Model of Landscape Preference. Leibniz International Proceedings in Informatics, LIPIcs 86, 19 (2017), 1-13. https://doi.org/10.4230/LIPIcs.COSIT.2017.19

[4] Paul Coulton, Haider Ali Akmal, and Joseph Lindley. 2020. Design Research and Object-Oriented Ontology. Open Philosophy 3, 1 (2020), $11-41$. https://doi.org/10.1515/opphil-2020-0002

[5] Paul Dourish. 2006. Re-Space-Ing Place: "Place" and "Space" Ten Years On. In Proceedings of the 2006 20th Anniversary Conference on Computer Supported Cooperative Work (Banff, Alberta, Canada) (CSCW '06). ACM, New York, NY, USA, 299-308. https://doi.org/10.1145/1180875.1180921

[6] Anthony Dunne and Fiona Raby. 2013. Speculative everything: Design, fiction, and social dreaming. The MIT Press, Cambridge, MA. https: //doi.org/10.1093/jdh/epv001

[7] Jan Gehl. 2010. Cities for people. Island Press, Washington.

[8] Alix Gerber. 2018. Participatory speculation: Futures of public safety. In ACM International Conference Proceeding Series, Vol. 2. Association for Computing Machinery, New York, New York, USA, 1-4. https://doi.org/10.1145/3210604.3210640

[9] Edward Twitchell Hall. 1969. The hidden dimension: man's use of space in public and private. Doubleday, New York. xii, 217 sivua, [16] kuvasivua pages. https://oula.finna.fi/Record/oula.328721

[10] Steve Harrison and Paul Dourish. 1996. Re-Place-Ing Space: The Roles of Place and Space in Collaborative Systems. In Proceedings of the 1996 ACM Conference on Computer Supported Cooperative Work (Boston, Massachusetts, USA) (CSCW '96). ACM, New York, NY, USA, 67-76. https://doi.org/10.1145/240080.240193

[11] B Hillier. 2007. Space is the Machine. A configurational Theory. Cambridge University Press, Cambridge University Press, University Printing House, Shaftesbury Road, Cambridge, CB2 8BS, United Kingdom. http://discovery.ucl.ac.uk/49843/

[12] Yen-Chia Hsu, Jennifer Cross, Paul Dille, Michael Tasota, Beatrice Dias, Randy Sargent, Ting-Hao (Kenneth) Huang, and Illah Nourbakhsh. 2019. Smell Pittsburgh: Community-Empowered Mobile Smell Reporting System. In Proceedings of the 24th International Conference on Intelligent User Interfaces (Marina del Ray, California) (IUI '19). ACM, New York, NY, USA, 65-79. https://doi.org/10.1145/3301275.3302293

[13] Andrew Jenkins, Arie Croitoru, Andrew T. Crooks, and Anthony Stefanidis. 2016. Crowdsourcing a Collective Sense of Place. PLoS ONE 11, 4 (apr 2016), 1-20. https://doi.org/10.1371/journal.pone.0152932

[14] David Kirsh. 2019. Do architects and designers think about interactivity differently? ACM Transactions on Computer-Human Interaction 26, 2 (apr 2019), 1-43. https://doi.org/10.1145/3301425

[15] R. Kitchin. 2018. The Realtimeness of Smart Cities. Tecnoscienza : Italian fournal of Science \& Technology Studies 8 (2018), 19-42.

[16] Peter Gall Krogh, Marianne Graves Petersen, Kenton O’Hara, and Jens Emil Groenbaek. 2017. Sensitizing Concepts for Socio-Spatial Literacy in HCI. In Proceedings of the 2017 CHI Conference on Human Factors in Computing Systems (Denver, Colorado, USA) (CHI '17). Association for Computing 
Machinery, New York, NY, USA, 6449-6460. https://doi.org/10.1145/3025453.3025756

[17] Konstantinos Papangelis, Melvin Metzger, Yiyang Sheng, Hai-Ning Liang, Alan Chamberlain, and Vassilis-Javed Khan. 2017. "Get Off My Lawn!": Starting to Understand Territoriality in Location Based Mobile Games. In Proceedings of the 2017 CHI Conference Extended Abstracts on Human Factors in Computing Systems (Denver, Colorado, USA) (CHI EA '17). Association for Computing Machinery, New York, NY, USA, $1955-1961$. https://doi.org/10.1145/3027063.3053154

[18] Matti Pouke, Jorge Goncalves, Denzil Ferreira, and Vassilis Kostakos. 2016. Practical simulation of virtual crowds using points of interest. Computers, Environment and Urban Systems 57 (2016), 118-129. https://doi.org/10.1016/j.compenvurbsys.2016.02.004

[19] Shenando Stals, Michael Smyth, and Oli Mival. 2019. UrbanixD: From ethnography to speculative design fictions for the hybrid city. In $A C M$ International Conference Proceeding Series. Association for Computing Machinery, New York, NY, USA, 1-10. https://doi.org/10.1145/3363384.3363486

[20] Niels van Berkel, Jorge Goncalves, Simo Hosio, and Vassilis Kostakos. 2017. Gamification of Mobile Experience Sampling Improves Data Quality and Quantity. Proc. ACM Interact. Mob. Wearable Ubiquitous Technol. 1, 3, Article 107 (Sept. 2017), 21 pages. https://doi.org/10.1145/3130972 